\section{Silver Particles Enhance Emission of Fluorescent DNA Oligomers}

BioTechniques 34:62-68 (January 2003)

\section{ABSTRACT}

Here we describe a new opportunity in methodology for increasing the detectability of fluorescently labeled DNA on solid substrates. We show that the use of glass substrates coated with metallic silver particles results in an approximate 5-fold increase in the intensity of Cy3-or Cy5-labeled DNA oligomers. Proximity to these silver particles also increases the photostability of Cy3- and Cy5-labeled oligomers. These results suggest the use of DNA array substrates with silver particles for increased sensitivity in genetic analysis.

\section{INTRODUCTION}

DNA arrays are widely used in studies of gene expression $(2,5,16)$. Detection of hybridized DNA is usually performed using fluorescently labeled DNA. Since the labeled DNA is only present on the surface, the fluorescent signal is limited. Methods that increase the total emission per labeled DNA molecule would provide increased sensitivity, a wider dynamic range, and fewer problems with unwanted background from the reagents, substrates, or optical components.

We suggest the use of metal-enhanced fluorescence to improve detectability on DNA arrays. Metal-enhanced fluorescence is related to surface-enhanced Raman scattering (SERS). In the absence of metal surfaces, Raman scattering is a weak phenomenon and thus difficult to accomplish with dilute samples. However, Raman signals can be dramatically enhanced by the interaction of the target molecules with rough metallic surfaces, typically roughened silver electrodes or irregular silver particles $(7,21)$. The phenomenon of SERS increases the scattering intensity by a factor of $10^{6}$ for averaged signal and by factors as high as $10^{14}$ for individual molecules on selected metallic particles $(8,19)$. The SERS effect appears to be due to both the electromagnetic interactions of the molecules with the conducting surface and the specific interactions of surface-adsorbed molecules $(4,22)$.

Electromagnetic interactions also occur between fluorophores and conducting metallic surfaces and particles $(1,3,10)$. In the case of fluorescence, the molecules adsorbed directly on the surface are thought to be quenched. Thus, through space, electromagnetic interactions between fluorophores and the metallic surface (metal) are likely to be the origin of fluorescence spectral changes. While theoretical publications often focus on SERS, these same papers often describe the predicted effects on fluorescence. A wide range of effects are expected, including increased and decreased quantum yields, increased and decreased lifetimes, changes in photostability, and increased distances for resonance energy transfer (RET). Given the ubiquitous use of fluorescence in biotechnology and DNA analysis, we have investigated the effects of silver particles on a variety of fluorophores and RET (12-15).
In this report, we focused on whether metallic silver particles can cause useful spectral changes for labeled DNA under the conditions used on DNA arrays. In our experiment, we used dsDNA labeled with either Cy3 or Cy5 and tethered to amino-coated quartz slides, which were half covered with silver island films. Silver island films consist of a layer of sub-wavelength size silver particles that cover about $20 \%$ of the surface and do not form a continuous silver coating. These films display the plasmon resonance typical for colloidal metal (9) and have a blue-green color but are not reflective.

\section{MATERIALS AND METHODS}

\section{Sample Preparation}

The labeled oligomers containing $\mathrm{Cy} 3$ or Cy5 on the $5^{\prime}$ ends (Figure 1) were obtained from Synthetic Genetics (San Diego, CA, USA). The complementary unlabeled oligonucleotides were obtained from the Biopolymer Core Facility of the University of Maryland School of Medicine.

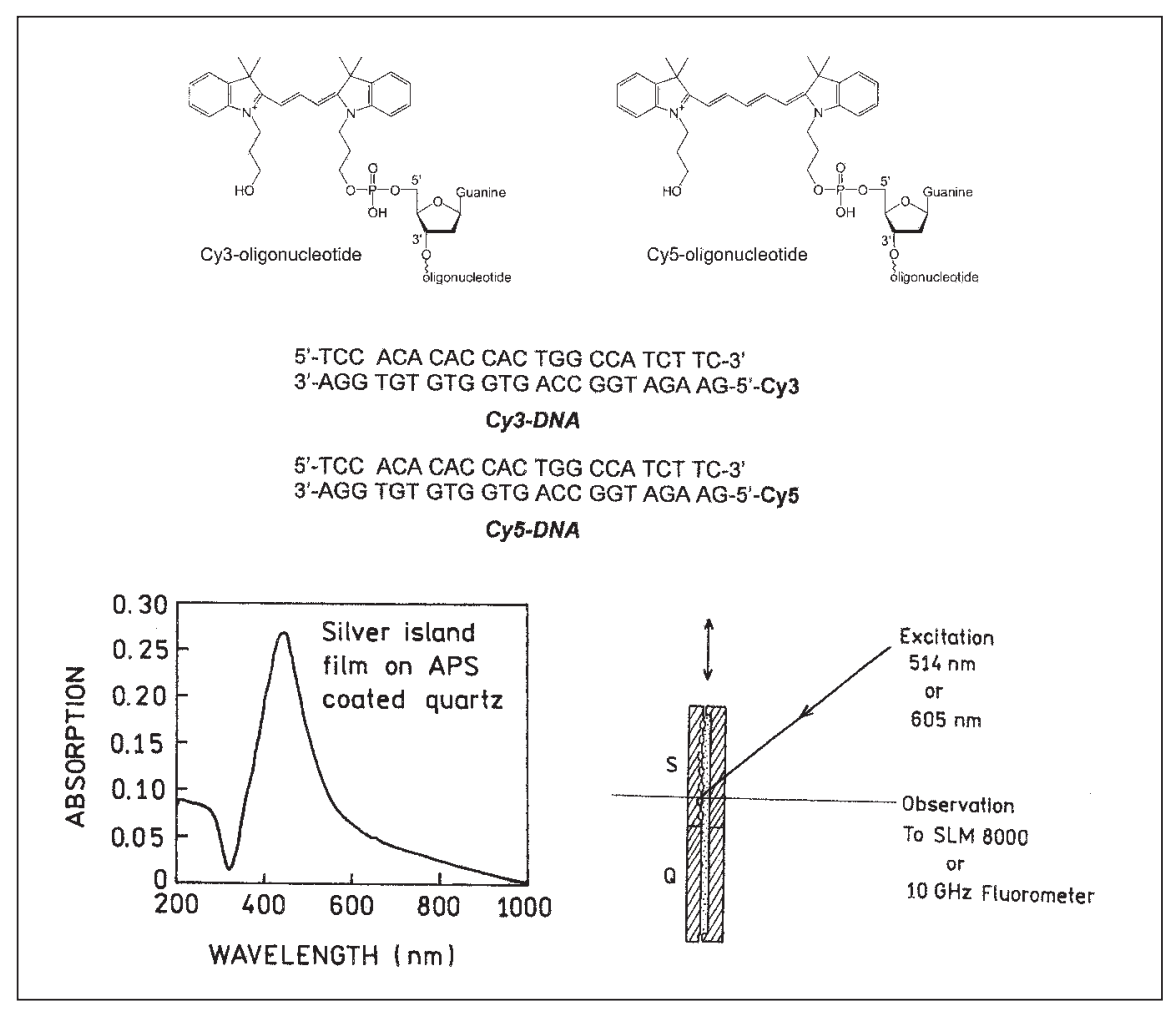

Figure 1. DNA structures and sample geometry. Structures and sequences of the labeled and unlabeled DNA oligomers (top). Absorption spectrum of silver islands on APS and experimental geometry (bottom). 
The dsDNA samples (Cy3-DNA or Cy5-DNA) were prepared by mixing the complementary oligonucleotides in $3 \times$ SSC buffer to a final concentration of $2 \mu \mathrm{M}$. The samples were then heated to $70^{\circ} \mathrm{C}$ for $2 \mathrm{~min}$, followed by slow cooling. Concentrations were determined using $\varepsilon(548 \mathrm{~nm})=150000 \mathrm{M}^{-1}$ $\mathrm{cm}^{-1}$ for Cy3 and $\varepsilon(648 \mathrm{~nm})=215000$ $\mathrm{M}^{-1} \mathrm{~cm}^{-1}$ for Cy5. The quantum yields were calculated using rhodamine $\mathrm{B}$ in water $(\mathrm{Q}=0.48)$ as a reference. The quantum yields of Cy3-DNA and Cy5DNA in the buffer solution were found to be 0.24 and 0.20 , respectively.

We used quartz slides to minimize background emission in our measurements. The entire surface of each slide $(1 \times 4 \mathrm{~cm})$ was coated with amino groups using 3-aminopropyltriethoxysilane (APS; Sigma, St. Louis, MO, USA). For this purpose, the slides were rigorously cleaned, soaked in a $0.1 \%$ aqueous solution of APS for $10 \mathrm{~min}$, and rinsed with water.

Silver island films were formed on half of the amino-coated slides, and the other half was left as an unsilvered control. Silver was deposited by the reduction of silver nitrate using D-glucose, as described previously $(13,18)$. The particles obtained were typically 100-300 $\mathrm{nm}$ across, $60 \mathrm{~nm}$ high, and covered about $20 \%$ of the treated surface. These islands displayed the typical surface plasmon resonance absorption with a peak near $440 \mathrm{~nm}$ (Figure 1).

Labeled dsDNA samples (Cy3-DNA or Cy5-DNA) previously hybridized in solution were deposited on the APStreated slides by placing $250 \mu \mathrm{L} 2 \mu \mathrm{M}$ solution on a $1 \times 4 \mathrm{~cm}$ area. The slides were illuminated with a shortwave UV lamp (model UVGL; $25 \mathrm{~W}$ for $15 \mathrm{~min}$ at a distance of $3 \mathrm{~cm}$ ) to provide DNA crosslinking to the amino-coated surface and then washed extensively with buffer. The DNA-coated surface was maintained in buffer by using a $0.2-\mathrm{mm}$ demountable cuvette (Starna Cell, Atascadero, CA, USA) that was not coated with amino groups or silver. We attempted the absorption measurements of $\mathrm{Cy} 3$ DNA and Cy5-DNA on the unsilvered and silvered areas. We did not notice any significant (more than 20\%) difference in dye absorptions, and we believe that the surface concentration of the tethered probes did not differ more than $20 \%$ on the silvered and unsilvered areas.

\section{Fluorescence Measurements}

For steady-state and time-resolved fluorescence measurements, the sample was placed diagonally with an approxi-

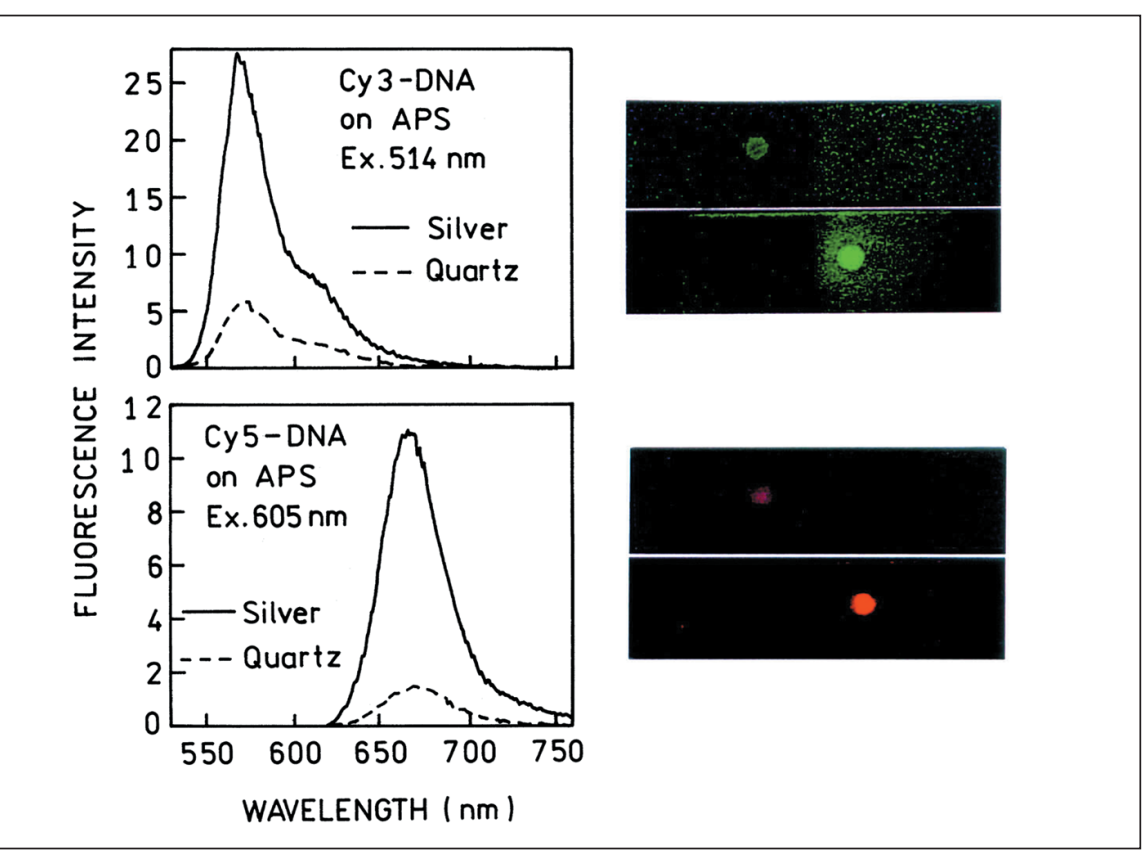

Figure 2. Emission spectra of Cy3-DNA (top) and of Cy5-DNA (bottom) on APS-treated slides, with and without silver island films. The photograph shows the fluorescence spots on quartz and silver taken through 530 and $630 \mathrm{~nm}$ long pass filters for Cy3-DNA and Cy5-DNA, respectively.

mate $45^{\circ}$ incident illumination and observation (Figure 1, bottom). Emission spectra were obtained using an SLM 8000 spectrofluorometer and 514 and $605 \mathrm{~nm}$ excitation for $\mathrm{Cy} 3$ and $\mathrm{Cy} 5$, respectively. The emission was selected using 530 and $630 \mathrm{~nm}$ long pass filters for Cy3 and Cy5, respectively. Lifetime measurements were performed on a 10$\mathrm{GHz}$ frequency-domain fluorometer (11) with picosecond resolution. For the Cy3-DNA sample measurement, the excitation source was a mode-locked argon ion laser, $514 \mathrm{~nm}$, at about $76 \mathrm{MHz}$ pulse repetition rate. For Cy5-DNA, we used a cavity-dumped R6G dye laser, $605 \mathrm{~nm}$, at $3.8 \mathrm{MHz}$ repetition rate. The same laser light sources were used for the steady-state and frequency-domain measurements and for photographs. The frequency-domain data were obtained with magic angle polarizer conditions. The emission of $\mathrm{Cy} 3$ was observed through a combination of a $565-\mathrm{nm}$ interference filter and a 530-nm long pass filter, and Cy5 was observed with a 665$\mathrm{nm}$ interference filter combined with a 630-nm long pass filter.

\section{RESULTS AND DISCUSSION}

Figure 2 shows the emission spectra of Cy3-DNA and Cy5-DNA on slides. Depending on the particular silvercoated slide, we observed 5- to 10-fold increases in intensity from the silvercoated part of the slide, as compared with the unsilvered part of the slide. Figure 2 also shows photographs that illustrate Cy3-DNA and Cy5-DNA emission on quartz and silver island films.

It is well known that the quantum yield of a fluorophore is given by

$$
Q_{0}=\frac{\Gamma}{\Gamma+k_{n r}}
$$

where $\Gamma$ and $\mathrm{k}_{\mathrm{nr}}$ are the radiative and non-radiative decay rates, respectively. The lifetime is given by

$$
\tau_{0}=\frac{1}{\Gamma+k_{n r}}
$$

Increases in fluorescence intensity are frequently observed because of the decreases in $\mathrm{k}_{\mathrm{nr}}$, which often occur when fluorophores are immobilized relative to the free solution. Therefore, 
one explanation for the effects seen in Figure 2 is a lower value of $k_{n r}$ on the unsilvered part of the slide. A decrease in $\mathrm{k}_{\mathrm{nr}}$ would result in an increase in the lifetime (Equation 2).

We measured the frequency-domain intensity decays of Cy3-DNA and Cy5DNA on the unsilvered and silvered regions of the slides (Figure 3). The lifetimes are dramatically shortened on the silvered part of the slides, which is better visualized on the time-domain representation of the decay data (Figure 3 and Table 1). An increase in intensity and a decrease in lifetime indicate an increase in the radiative decay rate $\Gamma$ to a larger value near the silver particles $\left(\Gamma_{\mathrm{m}}\right)$. Increases in radiative decay rates are expected for fluorophores at appropriate distances from silver particles $(1,3,10)$.

Our samples are spatially heterogeneous in that the labeled DNA is not localized to a specific distance from the silver surfaces and thus display a range of $\Gamma_{\mathrm{m}}$ values. Some oligomers may be located on the silver, adjacent to the silver-quartz interface or on the quartz distant from the silver. Nonetheless, it is of interest to estimate the increases in the radiative decay rate, which are consistent with our measurements. Examinations of Equations 1 and 2 show that the radiative decay rate is given by

$$
\frac{Q_{0}}{\tau_{0}}=\Gamma
$$

We calculated apparent values of $\Gamma$ using the intensities and lifetimes observed on the slides without $(\mathrm{O})$ and with $(-)$ silver. We assumed the quantum yields of Cy3-DNA and Cy5DNA, in the absence of silver, were the same as we observed previously in solution (17); that is, 0.24 and 0.20 , respectively. It is difficult to interpret the intensities on the silver-coated surfaces because the intensities are dependent on both an increased quantum yield of the fluorophores and increased excitation. The latter effect is due to an enhanced electric field near the particles caused by interactions between the free electrons in the metal and the incident light (10). With no consideration of this "lightening rod effect," the apparent quantum yields of Cy3 and Cy5 exceed unity on the silver surface. Since this is impossible, there must be some compo-
Table 1. Decay in Intensity of Emission from Cy3-DNA and Cy5-DNA on APS-Coated Quartz Slides

\begin{tabular}{|c|c|c|c|c|c|c|}
\hline Sample & $\bar{\tau}(\mathbf{n s})$ & $<\tau>$ (ns) & $\alpha_{i}$ & $f_{i}$ & $\tau_{\mathrm{i}}(\mathrm{ns})$ & $\chi_{\mathrm{R}}^{2}$ \\
\hline \multirow[t]{2}{*}{ Суз-DNA, Q } & $1.345^{\mathrm{a}}$ & $1.057^{b}$ & 0.362 & 0.111 & 0.325 & \\
\hline & & & 0.638 & 0.889 & 1.472 & 1.4 \\
\hline \multirow[t]{3}{*}{ Суз-DNA, S } & 0.532 & 0.125 & 0.674 & 0.151 & 0.028 & \\
\hline & & & 0.269 & 0.394 & 0.183 & \\
\hline & & & 0.057 & 0.455 & 1.003 & 1.9 \\
\hline \multirow[t]{2}{*}{ Cy5-DNA, Q } & 1.560 & 0.833 & 0.614 & 0.159 & 0.215 & \\
\hline & & & 0.386 & 0.841 & 1.815 & 1.4 \\
\hline \multirow[t]{3}{*}{ Cy5-DNA, S } & 0.411 & 0.041 & 0.923 & 0.438 & 0.019 & \\
\hline & & & 0.069 & 0.287 & 0.169 & \\
\hline & & & 0.008 & 0.275 & 1.353 & 1.6 \\
\hline \multicolumn{7}{|c|}{$\begin{array}{l}\text { Q, quartz; S, silver. } \\
\text { a } \bar{\tau}=\Sigma f_{i} \tau_{i} \text { where } f_{i}=\alpha_{i} \tau_{i} / \Sigma \alpha_{i} \tau_{i} \\
\text { b }<\tau>=\Sigma \alpha_{\mathrm{i}} \tau_{\mathrm{i}}\end{array}$} \\
\hline
\end{tabular}

nent of the enhanced field effect that contributes to the increased intensities in Figure 2. To estimate $\Gamma_{\mathrm{m}}$, we assumed the quantum yield on the silver surface increases by $1 / \mathrm{Q}_{0}(10)$, which is the largest possible value and implies that $\mathrm{Q}_{\mathrm{m}}$ is unity.

The intensity decays are heterogeneous and described by the multiexponential model

$$
I(t)=\sum \alpha_{i} \exp \left(-t / \tau_{i}\right)
$$

where $\alpha_{i}$ is the amplitude of the component with a lifetime $\tau_{i}$, and $\Sigma \alpha_{i}=$ 1.0. In solution, Cy3-DNA intensity decay can be approximated with two life- times: $\tau_{1}=0.38 \mathrm{~ns}$ and $\tau_{2}=1.33 \mathrm{~ns}$ with amplitudes $\alpha_{1}=0.623$ and $\alpha_{2}=$ 0.377. For Cy5-DNA, a good fit is achieved with $\tau_{1}=0.43 \mathrm{~ns}$ and $\tau_{2}=$ 1.44 ns with amplitudes $\alpha_{1}=0.471$ and $\alpha_{2}=0.529$. The average lifetimes in solution are slightly shorter than on quartz (Table 1). We believe that this is an effect of more rigid environment on quartz than in solution; that is, some non-radiative deactivation via rotations of the fluorophore is hindered on the surface.

While the lifetimes on the unsilvered area are only slightly nonexponential $(11 \%$ and $16 \%$ for the short

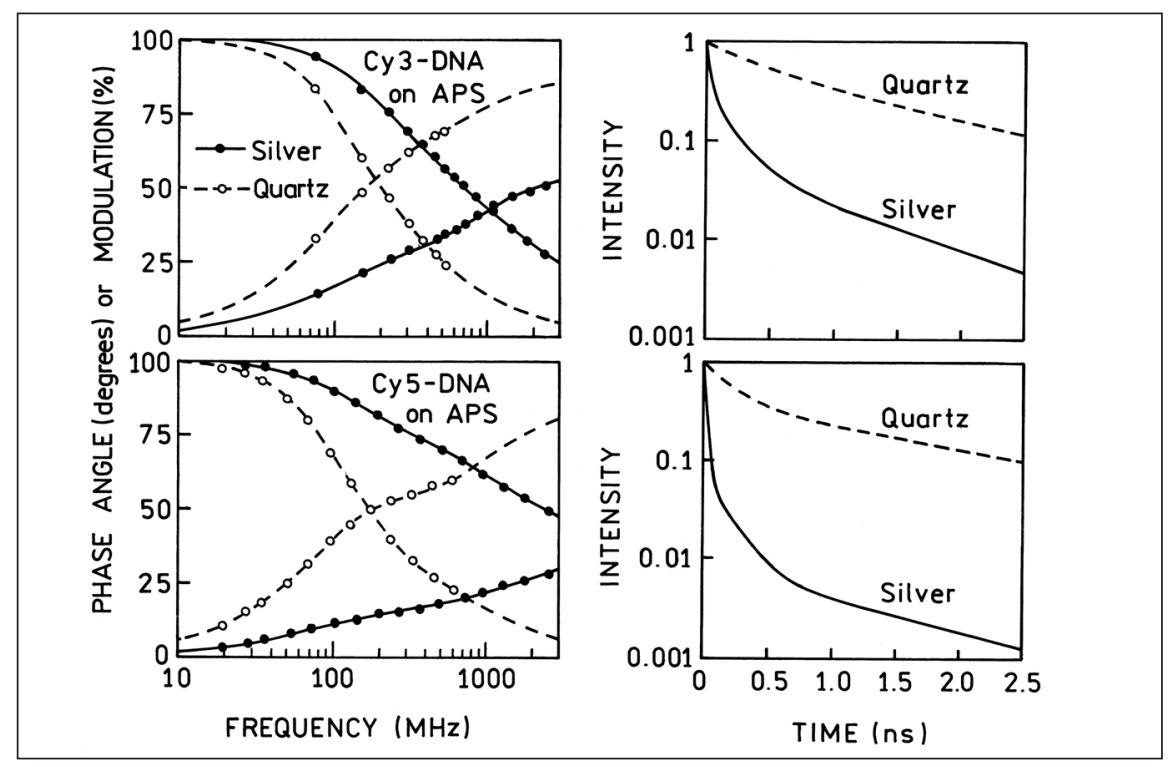

Figure 3. Frequency-domain intensity decays of Cy3-DNA and Cy5-DNA on APS-treated slides, with $(\Theta)$ and without $(0)$ silver island films. The right panels show the time-domain representation of the frequency-domain data. 
components), the lifetimes on the silvered areas are strongly heterogenous. We believe the multiexponential decays represent the range of possible distances between the probe and the surface. The frequency responses shift dramatically to higher frequencies (Figure 3), indicating shortened lifetimes. For the lifetime on the surfaces, we used the amplitude-weighted lifetime $<\tau>=\Sigma \alpha_{i} \tau_{i}$. Using these assumptions, we calculated the relative increase in the radiative decay rate $\Gamma_{\mathrm{m}} / \Gamma$. These calculations suggest that the radiative decay rates of Cy3-DNA and Cy5-DNA are increased 40- and 100fold, respectively, on the silver-coated part of the slides.

It is of interest to consider the increased signal that could be obtained using silver-coated surfaces. The total emission detectable from a fluorophore is usually limited by its photostability $(6,20)$. Photochemical degradationoccurs in the excited state so that a decreased lifetime should allow the fluorophore to undergo more excitationdeexcitation cycles before photobleaching. Therefore, we examined the emission intensity of Cy3-DNA and Cy5-DNA with continuous illumination (Figure 4). These traces of intensity versus time were obtained with the same incident intensity so that the time-zero values represent the increased intensities shown in Figure 2. If the time-zero values are normalized then the relative rates at which the emission decreases are about the same on the quartz or silver regions of the slides. This result means that the increased intensity found on the silvered slide is not obtained at the expense of more rapid photobleaching. The relative areas under these curves $\left(\mathrm{I}_{\mathrm{m}} / \mathrm{I}_{0}\right)$ are 4.8 and 6.5 for Cy3 and $\mathrm{Cy} 5$, respectively. In other words, in this experiment, it was possible to extract within 5 min 4.8 and 6.5 times more photons from silvered areas for Cy3-DNA and Cy5-DNA, respectively. Thus, the use of substrates coated with silver particles can result in a substantial increase in the signal observed from cyanine-labeled DNA. In Figure 4, we present photobleaching data with the excitation power adjusted to yield the same emissive photon flux at time zero. These data also show that the number of extracted photons within the time of measurements is higher on the silvered areas than on the quartz alone.

In this experiment, there was limited control of the distance between the metal and the fluorophores, and the fluorophores were probably present in areas outside the regions where enhanced emission was obtained. It is therefore likely that even larger increases in intensity from labeled DNA can be obtained with localization of the fluorophores at optimal distances from the metallic surfaces.

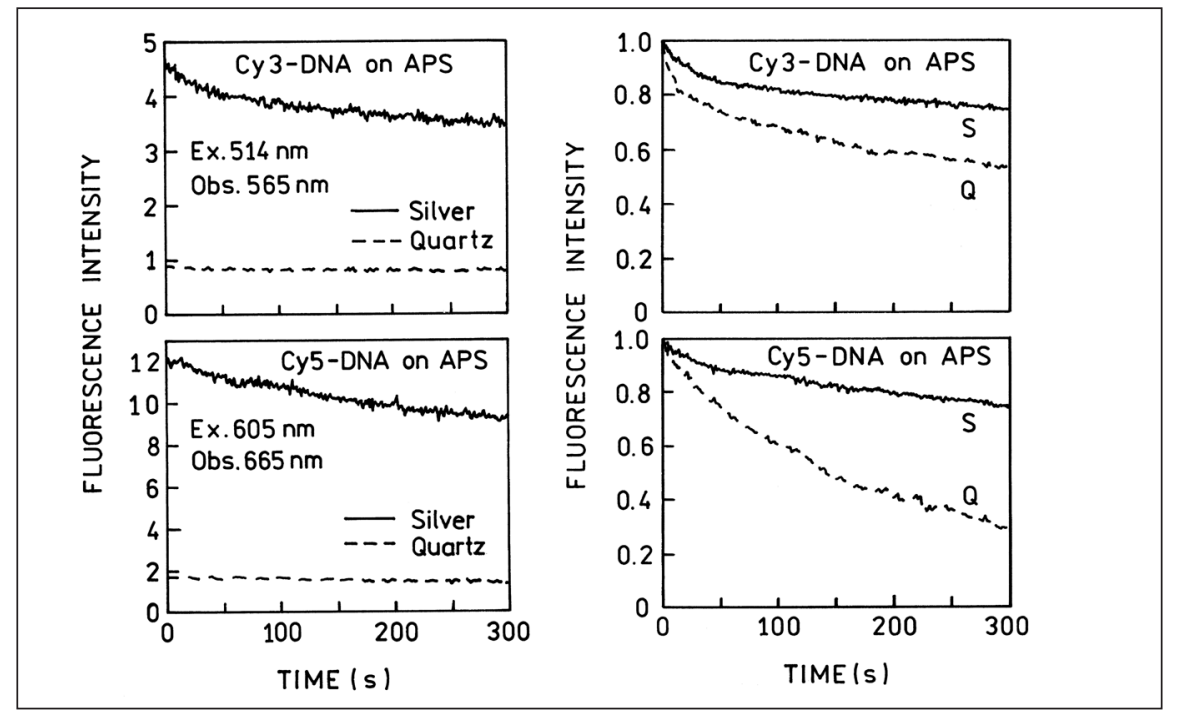

Figure 4. Photostability of Cy3-DNA and Cy5-DNA on APS-treated slides, with and without silver island films, for the same incident power (left), and the excitation intensity adjusted to yield the same emission intensities on quartz and silver (right).

\section{REFERENCES}

1.Barnes, W.L. 1998. Fluorescence near interfaces: the role of photonic mode density. J. Mod. Opt. 45:661-699.

2.Brown, P.O. and D. Botstein. 1999. Exploring the new world of the genome with DNA microarrays. Nat. Genet. Supp. 21:33-37.

3.Chance, R.R., A. Prock, and R. Silbey. 1978. Molecular fluorescence and energy transfer near interfaces. Adv. Chem. Phys. 37:1-65.

4.Chen, C.Y. and E. Burstein. 1980. Giant Raman scattering by molecules at metal-island films. Phys. Rev. Lett. 45:1287-1291.

5.Deyholos, M.K. and D.W. Galbraith. 2001. High-density microarrays for gene expression analysis. Cytometry 43:229-238.

6.Eggeling, C., J. Widengren, R. Rigler, and C.A.M. Seidel. 1999. Photostability of fluorescent dyes for single-molecule spectroscopy: mechanisms and experimental methods for estimating photobleaching in aqueous solutions, p. 562. In W. Rettig, B. Strehmel, S. Schrader, and H. Seifert (Eds.), Applied Fluorescence in Chemistry, Biology and Medicine. MAFS Proceedings Book, Springer-Verlag, New York.

7.Kneipp, K., H. Kneipp, I. Itzkan, R.R. Dasari, and M.S. Feld. 1999. Surface-enhanced Raman scattering: a new tool for biomedical spectroscopy. Curr. Sci. 77:915-924.

8.Kneipp, K., H. Kneipp, V. Bhaskaran Kartha, R. Manoharan, G. Deinum, I. Itzkan, R.R. Dasari, and M.S. Feld. 1998. Detection and identification of a single DNA base molecule using Surface-Enhanced Raman Scattering (SERS). Physical Rev. E 57:R6281-R6284.

9.Kreibig, U. and M. Vollmer. 1995. Optical Properties of Metal Clusters, Springer Series in Materials Science, p. 532. Springer-Verlag, Berlin.

10.Kummerlen, J., A. Leitner, H. Brunner, F.R. Aussenegg, and A. Wokaun. 1993. Enhanced dye fluorescence over silver island films: analysis of the distance dependence. Mol. Phys. 80:1031-1046.

11.Laczko, G., I. Gryczynski, Z. Gryczynski, W. Wiczk, H. Malak, and J.R. Lakowicz. 1990. A 10-GHz frequency-domain fluorometer. Rev. Sci. Instrum. 61:2331-2337.

12.Lakowicz, J.R. 2001. Radiative decay engineering: biophysical and biomedical applications. Anal. Biochem. 298:1-24.

13.Lakowicz, J.R., Y. Shen, S. D'Auria, J. Malicka, J. Fang, Z. Gryczynski, and I. Gryczynski. 2002. Radiative decay engineering: effects of silver island films on fluorescence intensity, lifetimes, and resonance energy transfer. Anal. Biochem. 301:261-277.

14.Lakowicz, J.R., Y. Shen, Z. Gryczynski, S. D'Auria, and I. Gryczynski. 2001. Intrinsic fluorescence from DNA can be enhanced by metallic particles. Biophys. Res. Commun. 286:875-879.

15.Lakowicz, J.R., J. Kusba, Y. Shen, J. Malicka, S. D'Auria, Z. Gryczynski, and I. Gryczynski. Effects of metallic silver particles on resonance energy transfer between fluorophores bound to DNA. J. Fluorescence (In Press.)

16.Lipshutz, R.J., S.P.A. Fodor, T.R. Gingeras, 
and D.J. Lockhart. 1999. High density synthetic oligonucleotide arrays. Nat. Genet. Supp. 1:20-24.

17.Malicka, J., I. Gryczynski, J. Fang, J. Kusba, and J.R. Lakowicz. Photostability of $\mathrm{Cy} 3$ and Cy5-labeled DNA in the presence of metallic silver particles. J. Fluorescence (In Press.)

18.Ni, F. and T.M. Cotton. 1986. Chemical procedure for preparing surface-enhanced Raman scattering active silver films. Anal. Chem. 58:3159-3163.

19.Nie, S. and S.R. Emory. 1997. Probing single molecules and single nanoparticles by surface-enhanced Raman scattering. Science 275:1102-1106

20.Soper, S.A., H.L. Nutter, R.A. Keller, L.M. Davis, and E.B. Shera. 1993. The photophysical constants of several fluorescent dyes pertaining to ultrasensitive fluorescence spectroscopy. Photochem. Photobiol. 57:972-977.

1.Vo-Dinh, T. 1998. Surface-enhance Raman spectroscopy using metallic nanostructures. Trends Anal. Chem. 17:557-582.

22.Wokaun, A. 1984. Surface-enhanced electromagnetic processes, pp 330. In H. Ehrenreich and D. Turnbull (Eds.), Solid State Physics: Advances in Research and Application. Vol. 38. Academic Press, New York.

This work was supported by the National Institutes of Health grant no. EB-00682 and the National Center for Research Resources grant no. RR-08119 to J.R.L. Address correspondence to Dr. Joseph Lakowicz, University of Maryland School of Medicine, Center for Fluorescence Spectroscopy, Department of Biochemistry and Molecular Biology, 725 West Lombard Street, Baltimore, MD 21201, USA. e-mail: lakowicz@cfs.umbi.umd.edu

Received 21 June 2002; accepted 16 October 2002

\section{Joseph R. Lakowicz, Joanna Malicka, and Ignacy Gryczynski \\ University of Maryland School of Medicine \\ Baltimore, MD, USA}

For reprints of this or any other article, contact Reprints@BioTechniques.com

\section{Molecular Beacon Sequence Design Algorithm}

BioTechniques 34:68-73 (January 2003)

\section{ABSTRACT}

A method based on Web-based tools is presented to design optimally functioning molecular beacons. Molecular beacons, fluorogenic hybridization probes, are a powerful tool for the rapid and specific detection of a particular nucleic acid sequence. However, their synthesis costs can be consider able. Since molecular beacon performance is based on its sequence, it is imperative to rationally design an optimal sequence before synthesis. The algorithm presented here uses simple Microsoft ${ }^{\circledR}$ Excel ${ }^{\circledR}$ formulas and macros to rank candidate sequences. This analysis is carried out using mfold structural predictions along with other free Web-based tools. For smaller laboratories where molecular beacons are not the focus of research, the public domain algorithm described here may be usefully employed to aid in molecular beacon design.

\section{INTRODUCTION}

This report describes a method to aid the design of molecular beacon sequences. Molecular beacons are a new optical tool that can be used to detect the presence of a specific DNA sequence in a mixture of targets. First demonstrated by Tyagi and Kramer (4), molecular beacons are fluorogenic oligonucleotide probes that signal hybridization with a complementary nucleic acid target (3). These DNA oligonucleotides contain a 5' fluorophore, a 3' quenching group, and 4-6 complementary bases on the $3^{\prime}$ and 5 " "stem" ends so that, unless the inner "loop" region hybridizes to a complementary nucleic acid, the fluorescence of the beacon in its hairpin configuration is quenched (Figure 1). When hybridized with a complementary oligonucleotide, the hairpin structure linearizes, distancing the fluorophore and quencher to yield fluorescence. The usefulness of molecular beacons to signal a hybridization event depends on their molecular structure. Therefore, each molecular beacon must be designed such that its sequence confers the correct conformation in the hairpin unhybridized state and the linear form when hybridized to the intended target.

While designing molecular beacons that function properly is more complex than the design of simple linear probes, the advantages of molecular beacons outweigh the difficulties in their proper design. Traditionally, linear oligonucleotide probes used to identify hybridization of a specific nucleic acid sequence are tagged with fluorescent or radioactive labels and hybridized to a sample. Unhybridized probes are washed away if the sample is anchored to a solid surface (e.g., blot or tissue), or they are digested if the target is in solution (e.g., PCR or FISH). Remaining probe is assumed to be hybridized to the target and is quantified. The post-processing steps in these methods can alter the true conditions of hybridization and thus prevent the technique from being used in real-time and in vivo.

An essential aspect of molecular beacons is that the beacon cannot have a target hybridized in the loop region and still maintain its duplex conformation in the stem region. The rigidity of the DNA helix prevents beacon-target hybrids from coexisting with stem hybrids. If designed correctly, then a perfectly matched beacon-target hybrid will be energetically more stable than the beacon hairpin conformation. In addition, a mismatched beacon-target hybrid will not open the hairpin of the stem because of its lower energetic stability. These features impart a high level of specificity for the exact target sequence.

In addition to specificity for the desired target sequence, optimal design of molecular beacons dictates that fluorescence quenching of the beacon in the absence of a target should only occur in the correct hairpin conformation so that the $5^{\prime}$ - and $3^{\prime}$-ends are in close proximity. While the sequence of the loop region is constrained to be complementary to the desired nucleic acid target, the stem region sequence can contain any possible combination of nucleotides. Therefore, it is useful to predict what conformations are most likely for a molecular beacon of a given sequence.

While one could in theory synthe- 